\title{
Clinical Profile and Response to First Line Treatment in Chronic Phase of Chronic Myeloid Leukaemia
}

\author{
Sivasubramaniam K. ${ }^{1}$, Latha K. V.S. ${ }^{2}$, Abhishek R. ${ }^{3}$, Niranjan V. ${ }^{4}$, \\ Tintu M.V. ${ }^{5}$, Jyothis P. Jose ${ }^{6}$, Rahul T.S.7, Senthilkumar E. ${ }^{8}$ \\ 1, 2, 4, 5, 6, 7,8 Department of Medical Oncology, Madras Medical College, Chennai, Tamilnadu, India. \\ ${ }^{3}$ Department of Haematology, Madras Medical College, Chennai, Tamilnadu, India.
}

\section{ABSTRACT}

\section{BACKGROUND}

Chronic Myeloid Leukaemia (CML) usually occurs in the elderly population and its incidence is higher in India. Chronic Phase (CP) is treated with tyrosine kinase inhibitors (TKI) as first line until progression. Stem cell transplant is usually not done in chronic phase and it is reserved for accelerated phase and blast crisis. The exact incidence and treatment data from various institutions in India is lacking.

\section{METHODS}

81 patients diagnosed with chronic phase CML in our institute over a period of 5 years were analysed from the master case records. Their clinical profile, lab parameters, response to treatment with Imatinib and side effects of treatment were analysed. Data was analysed using SPSS version 23.

\section{RESULTS}

Median age of patient $(\mathrm{n}=81$ ) was 44 years (range 31 - 71 years). Majority of patients presented in the $5^{\text {th }}$ decade $(43.2 \%)$. Left hypochondrial pain was the most common complaint (84\%) followed by fatigue (80.2\%). $72.8 \%$ patients had WBC count of more than a lakh, and thrombocytosis in present in $63 \%$. Median blast count was 1.8 \%. $18(22.2 \%), 52(64.2 \%)$ and $11(13.6 \%)$ patients $(\mathrm{n}=81)$ had low, intermediate and high Sokal index respectively. CHR was achieved in $87.7 \%$ of patients $(n=71)$ at 3 months. $92.6 \%$ (74 patients) achieved CHR at 6 months. Median time to achieve CHR was 39 days. 2.5 year Progression free Survival (PFS) was $67.9 \%$ and 2.5 year Overall Survival (OS) was $72.8 \%$ combining all 3 risk categories in Sokal index. Most common toxicity was anaemia and its seen in $39.5 \%$ (32 patients) followed by Thrombocytopenia seen in $30.9 \%$ (25 patients).

\section{CONCLUSIONS}

Imatinib showed good response with manageable side effects in chronic phase CML in our institute. It is cost effective especially in the low socioeconomic group.

\section{KEY WORDS}

Chronic Myeloid Leukaemia, CML, Imatinib, Side Effects, First Line Treatment, Complete Hematologic Response, Progression Free Survival, Overall Survival
Corresponding Author:

Dr. Latha K.V.S.,

Madras Medical College,

Park Town, Chennai - 600006,

Tamilnadu, India.

E-mail: kvslatha63@gmail.com

DOI: $10.14260 / j e m d s / 2020 / 868$

How to Cite This Article:

Sivasubramaniam K, Latha KVS, Abhishek $R$, et al. Clinical profile and response to first line treatment in chronic phase of chronic myeloid leukaemia. J Evolution Med Dent Sci 2020;9(52):3973-3976, DOI: $10.14260 / \mathrm{jemds} / 2020 / 868$

Submission 15-07-2020,

Peer Review 02-11-2020,

Acceptance 09-11-2020,

Published 28-12-2020.

Copyright (C) 2020 Sivasubramaniam K. et al. This is an open access article distributed under Creative Commons Attribution License [Attribution 4.0 International (CC BY 4.0)] 


\section{BACKGROUND}

Chronic myeloid leukaemia arises from genetic aberration in precursor haematopoietic stem cell leading to uncontrolled proliferation of myeloid cells. In initial phase, more number of differentiated myeloid cells are formed and hence disease looks quiescent. It usually affects people in their $5^{\text {th }}$ or $6^{\text {th }}$ decade and forms around 15 to $20 \%$ of all adult leukaemia. ${ }^{1}$ There is a slight male predominance of disease. ${ }^{2}$ Ionizing radiation has been found to be a risk factor. ${ }^{3}$ It belongs to the myeloproliferative group of disorder. The disease is caused due to fusion of two genes namely BCR gene located on chromosome 22 and ABL1 gene located on chromosome 9 . The reciprocal translocation of these two genes form $t$ (9:22) (q34; q11) known as Philadelphia chromosome. The BCR-ABL1 fusion gene forms BCR-ABL1 fusion product that has tyrosine kinase catalytic activity thereby forming unregulated production of myeloid cells. Apart from neutrophils, other myeloid lineages namely basophils and eosinophils are also usually elevated in this disease. CML was the first cancer to be consistently associated with a known and specific translocation. Ionizing radiation exposure is the only known risk factor so far. Familial predisposition though rare can be seen in some cases usually due to Philadelphia chromosome or JAK2 mutation that is transmitted in an autosomal dominant fashion. Though rare three phases of disease have been noted namely Chronic Phase (CP), Accelerated Phase (AP) and Blast Phase (BP) and around 90 - $95 \%$ of patients present in chronic phase at presentation. As the disease progresses from chronic phase to accelerated or blast crisis there is block in formation of differentiated cells and more number of blasts start circulating. There is rapid decline in platelet count also. Most of the patients (30-50\%) are diagnosed incidentally during evaluation for other conditions. ${ }^{4}$ Other presenting complaints can be fatigue, weight loss, fever, malaise, abdominal fullness due to splenomegaly, inappropriate sweating, etc. ${ }^{4}$ Diagnosis is by peripheral smear and bone marrow aspiration or biopsy and demonstration of Philadelphia chromosome by either conventional karyotyping ( $90 \%$ ) or FISH (Fluorescence In Situ Hybridization) or RT-PCR (Real-Time Reverse Transcription Polymerase) methods. ${ }^{5}$ Prognosis of CML patients depends upon age of the patient, initial spleen size, percentage of blast cells and platelet counts as described by Sokal Index and ELTS scoring system. Hasford score includes eosinophil and basophil count along with the other parameters used in Sokal and ELTS system. These scoring systems guide the clinicians to choose the specific generation of tyrosine kinase inhibitors based on the previous trials. In early days chemotherapeutic drugs like busulfan, hydroxyurea, and splenic irradiation were used as treatment with minimal response. Stem cell transplant was also used but with a lot of morbidity and mortality. In 2002 , the introduction of imatinib in the market for treatment of CML made a tremendous impact. ${ }^{6}$ and the number of bone marrow transplants done upfront for this disease came down dramatically. Imatinib is a tyrosine kinase inhibitor in ABL protooncogene. With the usage of imatinib more treatment response guides came into practice like Complete Haematologic Response (CHR), Cytogenetic Response (CR) and Molecular Response (MR). Problem during drug therapy is side effects of imatinib and drug compliance and most of the patients will ultimately progress to AP or BP. ${ }^{7}$

\section{METHODS}

This is a descriptive study. Patients diagnosed with CML from January 2013 to December 2017 were searched from our medical records. A total of 97 patients were found, among whom 88 were in chronic phase at the time of diagnosis. Among them 7 patients who did not receive continuous treatment or lost for follow up were excluded and hence 81 patients were analysed. Details including age, sex, presenting complaints, their duration, clinical examination findings, lab results, Sokal index, general condition of patient and comorbidities were noted down and analysed.

\section{Statistical Analysis}

SPSS version 23 software was used for statistical analysis including descriptive study, response rate to imatinib, and side effect profile with imatinib. Mean, median, mode, range, percentages, response rate are all calculated using the software.

\section{RESULTS}

Out of 97 patients $90.7 \%$ (88 patients) presented in chronic phase. Among them 81 patients who were started on imatinib mesylate were analysed in this manuscript. Median age of patient ( $\mathrm{n}=81$ ) was 44 years (range $31-71$ years). Since our department deals with adult patient's paediatric CML were not registered. 49 (60.5\%) were male and 32 (39.5\%) were female. Table 1 shows number of patients in each age-group and majority of patients were in their $5^{\text {th }}$ decade $(43.2 \%)$ and $16 \%$ of patients presented in their $4^{\text {th }}$ decade. Nearly $80 \%$ of patients were symptomatic at the time of presentation with fatigue and left hypochondrial pain being the most common presentation with a median duration of 7.5 months (range 6.5 - 8.5) combined for both these symptoms. (Table 2). Only 18.5 $\%(\mathrm{n}=15)$ of patients were diagnosed incidentally. Splenomegaly was present in $86.4 \%(n=70)$ of patients while massive splenomegaly was present in $49.4 \%(n=40)$ of patients. $32.1 \%(n=26)$ patients had fever, $7.4 \%(n=6)$ patients had hepatomegaly $39.5 \%(n=32)$ patients had pallor and bleeding was very rare at presentation.

Lab parameters of all the patients are listed in Table 3 . White Blood Cells (WBC) count was more than a lakh in 72.8 $\%$ ( $\mathrm{n}-59$ ) of patients. Mild anaemia was present in $22.2 \%$ (n $=18$ ) of patients and moderate anaemia in $16 \%$ of patients. Most of them were normocytic and normochromic. Thrombocytosis was present in $63 \%$ of patients and more than 6 lakh cells was present in $46.9 \%$ of patients $(n=38)$. Basophilia was present in more than $95 \%$ of patients. Myeloblast count was less than $2 \%$ in $83.9 \%(n=68)$ of patients with a median blast count of $1.8 \%$ (range $0.5-8 \%$ ). Initial diagnosis was done by karyotyping in majority of the patients (96.3\%) and remaining were diagnosed by Fluorescent in situ hybridization. After diagnosis patients were classified according to Sokal index score as low $(<0.8)$, 
intermediate $(0.8-1.2)$ and high risk $(>1.2)$ to know the prognosis of the patient. 18 (22.2\%), 52 (64.2\%) and 11 (13.6 $\%)$ patients $(\mathrm{n}=81)$ had low, intermediate and high Sokal index respectively. All of them were started on imatinib 400 mg according to institutional protocol based on its long-term result from IRIS study. ${ }^{8}$

Monthly history and physical examination, complete blood count and spleen size were noted. Adherence to imatinib therapy was strictly monitored. Complete Haematological Response (CHR) was aimed after treatment initiation which includes disappearance of symptoms, normal spleen size, disappearance of blasts in peripheral smear, normal basophil count and platelet count less than 4.5 lakhs. CHR was achieved in $87.7 \%$ of patients $(n=71)$ at 3 months. Among 10 patients (12.3\%) who did not achieve CHR, 4 patients (4.9\%) were found to have poor drug compliance in-spite of initial counselling. They were again counselled and 3 patients achieved CHR in a month and the other one after 2 months. $92.6 \%$ (74 patients) achieved CHR at 6 months. Cytogenic and molecular response assessment were done if patients are affordable at 6 months or whenever there is haematological failure. Median time to achieve CHR was 39 days. 2.5-year Progression Free Survival (PFS) was $67.9 \%$ and 2.5-year Overall Survival (OS) was $72.8 \%$ combining all 3 risk categories in Sokal index.

Most of the patients tolerated imatinib well with side effects ranging from 20 - $40 \%$. Haematological toxicities were more common than non-haematological. Most common toxicity was anaemia which is seen in $39.5 \%$ (32 patients) with only $10 \%$ requiring blood transfusion. Thrombocytopenia was seen in $30.9 \%$ (25 patients) and most of them recovered within 1 - 2 weeks after withholding the drug. Neutropenia was present in $18.5 \%$ (15 patients). Pedal oedema was the most common non haematological toxicity seen in $25.9 \%$ patients followed by skin changes in the form of hypo or hyperpigmentation in $23.5 \%$ of patients. Musculoskeletal pain was present in $22.2 \%$ of patients $(\mathrm{n}=$ 18) and diarrhoea usually grade $1-2$ in $11.1 \%$ of patients at some point of time and managed conservatively. Elevated Liver Function Test (LFT) was present in 9 patients $(11.1 \%)$ and no one developed more than five times the upper limit of liver enzymes.

\begin{tabular}{|cc|}
\hline Age in Years & Number (\%) $\mathbf{n}=\mathbf{8 1}$ \\
$<30$ & - \\
$30-40$ & $13(16)$ \\
$40-50$ & $35(43.2)$ \\
$50-60$ & $24(29.2)$ \\
$60-70$ & $7(8.6)$ \\
$>70$ & $2(2.5)$ \\
\hline \multicolumn{2}{|c|}{ Table 1. Age Distribution } \\
\hline
\end{tabular}

\begin{tabular}{|ccc|}
\hline Symptom / Sign & Number (\%) & $\begin{array}{c}\text { Median Duration in } \\
\text { Months }\end{array}$ \\
Asymptomatic & $15(18.5)$ & - \\
Fatigue & $65(80.2)$ & 8.5 \\
Left hypochondrial Pain / Fullness & $68(84)$ & 6.5 \\
Fever & $26(32.1)$ & 0.5 \\
Splenomegaly & $70(86.4)$ & \\
$<5 \mathrm{~cm}$ & $11(13.5)$ & \\
$5-8 \mathrm{~cm}$ & $19(23.5)$ & - \\
$>8 \mathrm{~cm}$ & $40(49.4)$ & - \\
Hepatomegaly & $6(7.4)$ & - \\
Pallor & $32(39.5)$ & - \\
Bleeding & $1(1.2)$ & \\
Lymphadenopathy & $4(4.9)$ \\
\hline \multicolumn{2}{c}{ Table 2. Presentation } \\
\hline \multicolumn{2}{c}{}
\end{tabular}

\begin{tabular}{|c|c|}
\hline Lab Parameter & Number $(\%)$ n = 81 \\
\hline WBC Count (cells / $\mu \mathrm{l}$ ) & $81(100)$ \\
\hline$<50,000$ & $8(9.9)$ \\
\hline $50,000-1,00,000$ & $14(17.3)$ \\
\hline$>1,00,000$ & $59(72.8)$ \\
\hline \multicolumn{2}{|l|}{ Haemoglobin (gm / dl) } \\
\hline$<8$ & $7(8.6)$ \\
\hline $8-10$ & $13(16)$ \\
\hline $10-12$ & $18(22.2)$ \\
\hline \multicolumn{2}{|l|}{ Platelet (Lakhs Cells / $\mu$ ) } \\
\hline $4.5-6$ & $13(16.04)$ \\
\hline$>6$ & $38(46.9)$ \\
\hline Basophilia $(>200$ cells $/ \mu \mathrm{l}$ ) & $79(97.5)$ \\
\hline \multicolumn{2}{|l|}{ Blast \% in PS or Marrow } \\
\hline$<2$ & $68(83.9)$ \\
\hline $2-5$ & $9(11.1)$ \\
\hline $5-10$ & $4(4.9)$ \\
\hline \multicolumn{2}{|l|}{ Philadelphia Chromosome } \\
\hline Conventional Cytogenetics & $78(96.3)$ \\
\hline FISH & $3(3.7)$ \\
\hline \multicolumn{2}{|c|}{ Table 3. Lab Paramet } \\
\hline
\end{tabular}

\begin{tabular}{|cc|}
\hline Adverse Effects & Number (\%) \\
Anaemia & $32(39.5)$ \\
Neutropenia & $15(18.5)$ \\
Thrombocytopenia & $25(30.9)$ \\
Skin Changes & $19(23.5)$ \\
Pedal Oedema & $21(25.9)$ \\
Abnormal LFT & $11(13.6)$ \\
musculoskeletal Pain & $18(22.2)$ \\
Diarrhoea & $9(11.1)$ \\
\hline \multicolumn{2}{|c|}{ Table 4. Adverse Effects } \\
\hline
\end{tabular}

\section{DISCUSSION}

There was slightly increase in number of patients presented in chronic phase of CML (90.7\%) compared to that given in literature (around $85 \%$ ). ${ }^{3}$ Median age of the patient was also earlier (44 years) but in West and European countries it is usually between 50 - 70 years. ${ }^{9-11}$ The reason behind early age of presentation is not known and will need special attention. Male predominance was also seen in our study population. Majority of the patients were symptomatic (80\%) while in other studies it was less than $50 \%{ }^{4}$ which can be correlated with ignorance of patients. More patients had fatigue (80.2\%) and left hypochondrial pain (84\%) which reflects advanced disease load and in turn reflects poor treatment outcome. The median duration of these symptoms is 7.5 months and it tells us the need for these patients to be suspected and referred early from primary and secondary care centres who initially see the bulk of the patients presenting here. Most of the times early symptoms which are nonspecific are neglected both by the patient and the initial treating physician. Patients diagnosed in asymptomatic stage are very less (18.5\%) whereas its 20 - $50 \%$ in western literature. ${ }^{3,4}$ Splenomegaly also is very high compared to that given in Western literature ${ }^{4}$ and patient experiencing any form of discomfort due to splenomegaly like early satiety, dull aching hypochondrial pain, and abdominal distention which is the trigger to visit hospital in many patients. Anaemia was not a troublesome issue during presentation since only $8.6 \%$ of patients had severe anaemia requiring blood transfusion. Anaemia can be due to various reasons that includes nutritional deficiency, splenomegaly and disease. $72.8 \%$ of patients were having WBCs more than a lakh at the time of presentation which makes us to treat initially with xanthine oxidase inhibitors and hydroxyurea in some patients due to the fear of tumour lysis 
syndrome although it is not done universally. Most of the patients $(83.9 \%)$ had less than $2 \%$ blasts in peripheral smear and bone marrow and thereby give better response to TKI although other factors increase the overall Sokal index of the patient. Most of our patients (64.2\%) had intermediate Sokal index and $13.6 \%$ of them had high risk score which suggests advanced disease. Imatinib was the first TKI used in CML patients and it has a very long term follow up results. Other advantage of imatinib is that it is less cost than second generation TKIs. Due to this various advantages imatinib is used in front line setting for all risk categories in our patients unless contraindicated or poorly tolerated. Complete Haematological Response (CHR) denotes disappearance of blasts in peripheral smear, basophils and WBC count normalizes and spleen becomes normal size. $87.7 \%$ of patients had CHR which gives symptom relief to patients especially fatigue, hypochondrial pain and dullness. Oral formulation of the drug also makes it patient friendly and easy to administer. Due to financial constraints to conduct molecular and cytogenetic response tests, they are done less frequently than recommended in our set up and it is reserved for conditions like response failure or whenever drug resistance is suspected along with imatinib resistance mutation analysis. $67.9 \%$ patients had PFS and $72.8 \%$ patients had OS at the end of 2.5 year follow up which gives a reasonable result and avoids the burden of haematopoietic stem cell transplant in these patients. Long term results of IRIS study showed 10 year OS of $83.3 \%$ and $82.8 \%$ of patients had complete cytogenetic response. ${ }^{8}$ Dose of imatinib is given as $400 \mathrm{mg}$ based on the previous results from studies that used $600 \mathrm{mg}$ and $800 \mathrm{mg}$ strengths that did not increase overall survival but produced more toxicity with higher doses.12-14 Side effect profile is favorable and most of them had haematological toxicity especially anaemia (39.5\%) and thrombocytopenia (30.9\%) which can be managed by withholding drug for 1 to 2 weeks. Skin changes, pedal oedema, myalgia and GI (Gastro-Intestinal) disturbances were present in less than $25 \%$ of patients and were not a worrying issue. LFT though deranges in $13.6 \%$ of patients most of them had elevated liver enzymes less than 3 - 5 times that of normal upper limit and recovered after withholding the drug and reducing the dose to $300 \mathrm{mg}$.

\section{CONCLUSIONS}

The milestones achieved in treatment of CML are numerous, but the worrisome part is that most of the patients will ultimately land up in AP or BP. Since India is a developing country, most of the centres use first generation TKI namely Imatinib, and second line TKIs are reserved for treatment failure and resistance to Imatinib.

Data sharing statement provided by the authors is available with the full text of this article at jemds.com.

Financial or other competing interests: None.

Disclosure forms provided by the authors are available with the full text of this article at jemds.com.

\section{REFERENCES}

[1] Siegel RL, Miller KD, Jemal A. Cancer Statistics, 2017. CA Cancer J Clin 2017;67(1):7-30.

[2] Chen $\mathrm{Y}$, Wang $\mathrm{H}$, Kantarjian $\mathrm{H}$, et al. Trends in chronic myeloid leukemia incidence and survival in the United States from 1975 to 2009 . Leuk Lymphoma 2013;54(7):1411-7.

[3] Faderl S, Talpaz M, Estrov Z, et al. The biology of chronic myeloid leukemia. N Engl J Med 1999;341(3):164-72.

[4] Savage DG, Szydlo RM, Goldman JM. Clinical features at diagnosis in 430 patients with chronic myeloid leukaemia seen at a referral centre over a 16-year period. $\mathrm{Br} \mathrm{J}$ Haematol 1997;96(1):111-6.

[5] Arber DA, Orazi A, Hasserjian R, et al. The 2016 revision to the World Health Organization classification of myeloid neoplasms and acute leukemia. Blood 2016;127(20):2391-405.

[6] Cortes J. Natural history and staging of chronic myelogenous leukemia. Hematol Oncol Clin North Am 2004;18(3):569-84.

[7] Druker BJ, Guilhot F, O'Brien SG, et al. Five-year follow-up of patients receiving imatinib for chronic myeloid leukemia. N Engl J Med 2006;355(23):2408-17.

[8] Hochhaus A, Larson RA, Guilhot F, et al. Long-term outcomes of imatinib treatment for chronic myeloid leukemia. N Engl J Med 2017;376(10):917-27.

[9] Sant M, Allemani C, Tereanu C, et al. Incidence of hematologic malignancies in Europe by morphologic subtype: results of the HAEMACARE project. Blood 2010;116(19):3724-34.

[10] Smith A, Howell D, Patmore R, et al. Incidence of haematological malignancy by sub-type: a report from the Haematological Malignancy Research Network. Br J Cancer 2011;105(11):1684-92.

[11] Chen Y, Wang H, Kantarjian $\mathrm{H}$, et al. Trends in chronic myeloid leukemia incidence and survival in the United States from 1975 to 2009 . Leuk Lymphoma 2013;54(7):1411-7.

[12] Baccarani M, Druker BJ, Branford S, et al. Long-term response to imatinib is not affected by the initial dose in patients with Philadelphia chromosome-positive chronic myeloid leukemia in chronic phase: final update from the Tyrosine Kinase Inhibitor Optimization and Selectivity (TOPS) study. Int J Hematol 2014;99(5):616-24.

[13] Deininger MW, Kopecky KJ, Radich JP, et al. Imatinib 800 mg daily induces deeper molecular responses than imatinib $400 \mathrm{mg}$ daily: results of SWOG S0325, an intergroup randomized PHASE II trial in newly diagnosed chronic phase chronic myeloid leukaemia. Br J Haematol 2014;164(2):223-32.

[14] Hehlmann R, Lauseker M, Saußele S, et al. Assessment of imatinib as first-line treatment of chronic myeloid leukemia: 10-year survival results of the randomized CML study IV and impact of non-CML determinants. Leukemia 2017;31(11):2398-406 